East African Medical Journal Vol. 86 No. 9 September 2009

PREGNANCY RATES AMONG FEMALE PARTICIPANTS IN PHASE I AND PHASE IIA AIDS VACCINE CLINICAL TRIALS IN KENYA

W. G. Jaoko, MBChB, MTMed, PhD, Associate Professor, Department of Medical Microbiology and Deputy Programme Director, Kenya AIDS Vaccine Initiative, University of Nairobi, P.O. Box 19676-00202, Nairobi, Kenya, H. Ogutu, KRN, BSc, Nurse/Counsellor, S. Wakasiaka, KRN, MPHE Nurse/Counsellor, R. Malogo, KRN, BA (Comm. Dev.) Nurse/Counsellor, R. Ndambuki, KRN, BA (Psych. Counselling) Nurse/Counsellor, J. Nyange, KRN, BA (Psych.), MSc (Clin. Psych), Clinical Trials Coordinator, G. Omosa-Manyonyi, MBChB, MSc (Infect. Dis.), Medical Manager, KAVI, University of Nairobi, P.O. Box 19676-00202, Nairobi, Kenya, P. Fast, MD, PhD, Chief Medical Officer, Research and Development, International AIDS Vaccine Initiative (IAVI), 110 William Street, 27th Floor, New York, NY 10038 - 3901 USA, C. Schmidt MD, MPH, DTMH, Medical Consultant, C. Verlinde, MSc, Director, Medical Affairs, International AIDS Vaccine Initiative, C. Smith MPH, Project Director, EMMES Corporation, 401 North Washington St., Suite 700, Rockville, MD 20850 USA, K.M. Bhatt, MBChB, MSc, MMed, Associate Professor, Department of Internal Medicine and Therapeutics, and Medical Consultant, KAVI, J. Ndinya-Achola, MBChB, MSc, Associate Professor, Department of Medical Microbiology and Consultant Advocacy Consultant, KAVI and O. Anzala, MBChB, PhD, Associate Professor, Department of Medical Microbiology and Programme Director, KAVI, College of Health Sciences, University of Nairobi, P. O. Box 19676-00202, Nairobi, Kenya

Request for reprints to: Prof. W. G. Jaoko, Kenya AIDS Vaccine Initiative, Department of Medical Microbiology, College of Health Sciences, University of Nairobi, P.O. Box 19676-00202, Nairobi, Kenya

\title{
PREGNANCY RATES AMONG FEMALE PARTICIPANTS IN PHASE I AND PHASE IIA AIDS VACCINE CLINICAL TRIALS IN KENYA
}

\author{
W. G. JAOKO, H. OGUTU, S. WAKASIAKA, R. MALOGO, R. NDAMBUKI, \\ J. NYANGE, G. OMOSA-MANYONYI, P. FAST, C. SCHMIDT, \\ C. VERLINDE, C. SMITH, K.M. BHATT, J. NDINYA-ACHOLA \\ and O. ANZALA
}

\begin{abstract}
Background: Female participants in AIDS candidate vaccine clinical trials must agree to use effective contraception to be enrolled into the studies, and for a specified period after vaccination, since the candidate vaccines' effects on the embryo or foetus are unknown.

Objectives: To review data on female participants' pregnancy rates from phase I and IIA AIDS vaccine clinical trials conducted at the Kenya AIDS Vaccine Initiative (KAVI) and to discuss the challenges of contraception among female participants.

Design: Descriptive observational retrospective study.

Setting: KAVI clinical trial site, Kenyatta National Hospital and University of Nairobi, Kenya.

Subjects: Thirty nine female participants were enrolled into these trials. They received family planning counselling and were offered a choice of different contraceptive methods, as per the protocols. All contraception methods chosen by the participants were offered at the study site at no cost to the participant.

Results: Four women conceived during the study period when pregnancies were to be avoided. All four had opted for sexual abstinence as a contraceptive method, but reported having been coerced by their partners to have unprotected sexual intercourse.

Conclusion: Abstinence is clearly not a reliable contraceptive option for women in developing-country settings. Effective female-controlled contraceptives, administered at the clinical trial site, may empower female participants to better control their fertility, leading to more complete clinical trial data.
\end{abstract}

\section{INTRODUCTION}

It is generally believed that a safe and efficacious preventive AIDS vaccine is one of the best longterm hopes to control and eventually eliminate the
HIV / AIDS pandemic (1). Of the 2.7 million new HIV infections that occur globally annually, more than half occur in women (2), and in many countries HIV prevalence among women has surpassed that among males in the sexually active age groups. 
HIV prevention among women faces many challenges. These challenges include the need for women to bear children; culturally defined gender roles and power dynamics that disempower women and constrain them from negotiating safe sex; lower economic and social power compared to men; and lack of access to health care, among others (3). The development of a preventive AIDS vaccine is therefore a high priority as a fully self-controlled preventive method.

Clinical trials testing AIDS candidate vaccines require the participation of both males and females in equitable numbers for several reasons (4). Firstly, researchers need to determine a candidate vaccine's safety and efficacy in both genders. As women and men are physiologically different, results and conclusions of vaccine efficacy and/or side effects from only one gender cannot be extrapolated to the other; indeed, recent findings suggest that HIV pathology may differ based on gender and route of infection (5). Secondly, licensure requires testing in fully representative populations. And thirdly, participants in AIDS vaccine research benefit from risk- reduction education, counselling and care; excluding women who are eligible to participate would not only be discriminatory, but would also be unethical based on the principle of equity $(3,4,6)$.

However, despite the importance of female participation in AIDS vaccine clinical trials, many researchers have, for diverse reasons, experienced difficulties recruiting women (4).

Female participants in AIDS vaccine clinical trials must use effective contraception during and for a period after vaccination, because the candidate vaccine's effects on the embryo or foetus are unknown. If a female participant conceives during the vaccination period, further vaccinations will not be given, which compromises the completeness of data and information gained from the trial. This circumstance further erodes the already diminished contribution of female participation in AIDS vaccine clinical trials. Every effort must therefore be made to ensure that female participants in such trials do not become pregnant during the specified period and that they are retained to the trial's conclusion.

Here, we present pregnancy data from four AIDS vaccineclinical trialsconducted since2001 at theKenya AIDS Vaccine Initiative (KAVI) research centre based at the University of Nairobi's School of Medicine, in Nairobi, Kenya. We make recommendations that would reduce the incidence of pregnancy in future clinical trials.

\section{MATERIALS AND METHODS}

Three Phase 1 trials were conducted between 2001 and 2004, testing (i) an HIV clade A plasmid DNA (DNA. HIVA) vaccine (Protocol IAVI 002), (ii) a recombinant vaccine vectored by modified vaccinia virus Ankara (MVA; MVA.HIVA) (IAVI 004), and (iii) DNA.HIVA as prime followed by MVA.HIVA as boost (IAVI 010). Phase IIA trial was conducted between 2005 and 2007 testing the VRC recombinant replication-defective Adenovirus sub-type 5-vectored multiclade vaccine (Vaccine Research Centre (VRC), NIH, VRC HIV-1 rAd5) either alone or as boost following VRC HIV-1 DNA vaccine (IAVI VRC V001). The Phase IIA trial was conducted prior to the outcome of theSTEP trial, a test-of-concept trial of a different Adenovirus subtype 5-vectored HIV vaccine developed by Merck Pharmaceuticals (7).

In all trials, participants were selected based on their self-reported low risk of HIV infection. For eligibility, participants were required to report that in the six months preceding enrolment they had not had unprotected vaginal or anal sex with a known HIV-infected person or a casual partner, had not engaged in sex work for money or drugs, had not used injection recreational drugs and had not had a sexually transmitted disease. To maintain confidentiality, the trial team did not inform participants' spouses about their participation in the trial. This responsibility was left to the discretion of the participants themselves.

Comprehensive family planning counselling, including contraceptive options, was discussed with all female participants in one-on-one sessions with trained nurse counsellors at every visit. Each female participant was then asked to select one contraceptive method of her choice, except in the first trial conducted in KAVI(IAVI002), in which all the female participants were required to use an oral contraceptive (Microgynon ${ }^{\circledR}$ ). The other contraceptive methods offered were the intrauterine contraceptive device (copper-T®), injectable hormonal contraceptive (Depo-provera ${ }^{\circledR}$ ), consistent male condom use and sexual abstinence. In all trials, the chosen family planning method was initiated at screening, which was up to six weeks before enrolment into the study. Based on screening results, participants were then enrolled into the trial. Family planning counselling sessions were conducted at each protocol visit.

Female participants were required not tobecome pregnant for specific periods: In the DNA.HIVA and the MVA.HIVA trials, this period was within four months after the last vaccination. In the DNA.HIVA prime/MVA.HIVA boost trial the period was within six months after the last vaccination, and in the VRC DNA prime/rAd5 boost trial, participants were prohibited from becoming pregnant for 18 months, that is, to the end of the trial.

Approvals to conduct the trials were obtained from Kenyatta National Hospital Ethics and Research Committee, Pharmacy and Poisons Board, University of Nairobi Institutional Biosafety Committee and National Council for Science and Technology. 


\section{RESULTS}

In total, 163 participants at self-reported low risk for HIV infection have participated in the four AIDS vaccine clinical trials conducted at KAVI since 2001, of whom 39 (23.9\%;) were females (Table 1$)$ aged between 18 and 48 years (median age 23 years).

Thirteen percent $(5 / 39)$ of the female participants had a university education, $74 \%(29 / 39)$ had secondary-level education (9-12 years of school), and $13 \%(5 / 39)$ had primary education (up to 8 years of formal school attendance). Thirty-one of the female participants were single, six were married and two were divorced.
Figure 1 and Table 1 show the distribution of contraceptive methods used by all the female participants during the four HIV vaccine trials at KAVI. The most commonly used methods of contraception were oral hormonal contraceptives $(31 \%)$, sexual abstinence $(26 \%)$ and injectable hormonal contraceptives (23\%). Table 2 shows the number of female participants who became pregnant in each of the trials. No participant in protocols IAVI 002 or IAVI 004 became pregnant during the period when pregnancy was not allowed according to the protocol. One of 12 participants in protocol IAVI 010 became pregnant, and three of the 22 female participants in protocol IAVI VRC V001 became pregnant. This

Table 1

Number of female participants in the four HIV vaccine clinical trials at the KAVI site, and contraceptive methods used

\begin{tabular}{|c|c|c|c|c|c|c|c|c|}
\hline Trial & $\begin{array}{l}\text { No. of female } \\
\text { volunteers/Total } \\
\text { No. of volunteers }\end{array}$ & OC & Norplant & IUCD & IHC & Abstinence & $\mathrm{MC}$ & Menopausal \\
\hline DNA（IAVI 002) & $3 / 18$ & 3 & 0 & 0 & 0 & 0 & 0 & 0 \\
\hline MVA (IAVI 004) & $2 / 18$ & 1 & 1 & 0 & 0 & 0 & 0 & 0 \\
\hline DNA prime/MVA & & & & & & & & \\
\hline boost (IAVI 010) & $12 / 70$ & 4 & 2 & 0 & 3 & 3 & 0 & 0 \\
\hline DNA prime/rAd5 & & & & & & & & \\
\hline boost (IAVI VRC V001) & $22 / 57$ & 4 & 1 & 1 & 6 & 7 & 2 & 1 \\
\hline Total & $39 / 166$ & 12 & 4 & 1 & 9 & 10 & 2 & 1 \\
\hline
\end{tabular}

$\mathrm{OC}=$ Oral contraceptives

IUCD = Intrauterine contraceptive devices

$\mathrm{IHC}=$ Injectable hormonal contraceptives

$\mathrm{MC}=$ Male condoms

Figure 1

Contraceptive methods used by all female participants in HIV vaccine clinical trials at KAVI, Nairobi
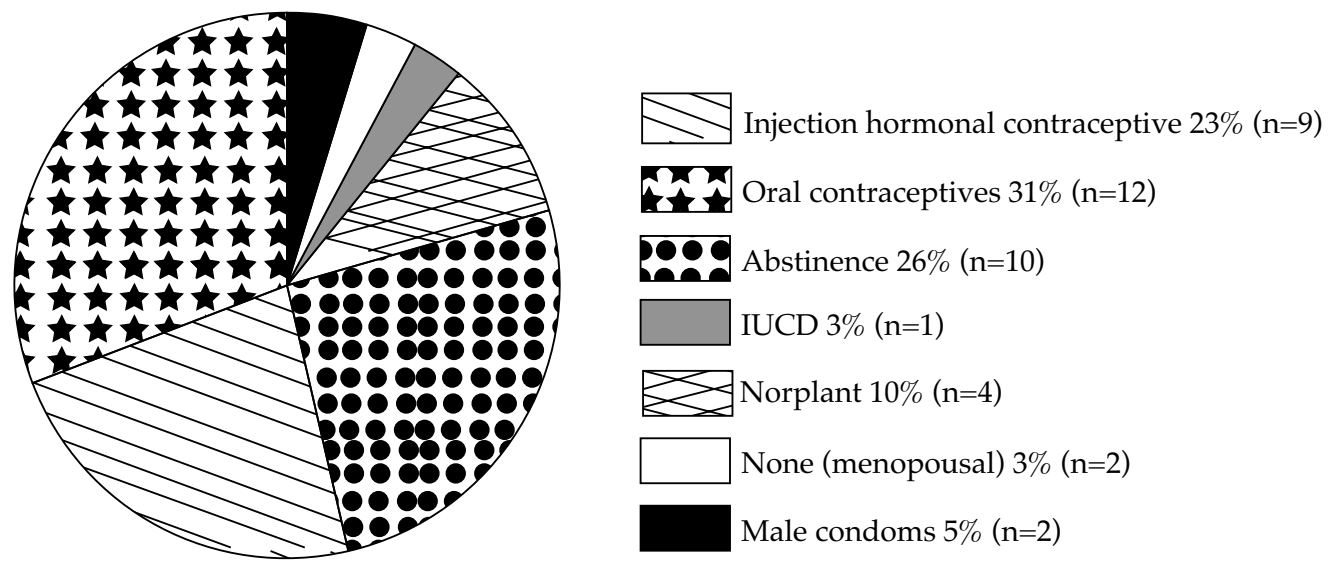
represents a pregnancy rate of 8.3 and $13.6 \%$ in trials with 12 and 22 female participants respectively. All the four female participants who became pregnant were single, with three of them being primigravida and the fourth having had one live birth with no history of miscarriages. All of these women had also chosen sexual abstinence as their contraception method. However, they all reported having been coerced by their partners to have unprotected sex. Table 2 also shows the period during the protocol in which the four participants became pregnant.

\section{DISCUSSION}

The participation of females in AIDS vaccine clinical trials testing is crucial, as it is the only way to determine the effects of candidate vaccines in women, and to detect any difference in the candidate vaccines' activity in males and females (4).

Recruiting female participants has been an ongoing challenge at our trial site; only $39(22 \%)$ women were enrolled out of the total of 163 participants in the four clinical trials conducted at KAVI since 2001. Although some studies have shown women's willingness to participate in AIDS vaccine clinical trials (8), minimal participation of women in such trials has been observed at other sites as well, and in all phases of HIV vaccine clinical trials (4).

Possible barriers specific to female participation in AIDS vaccine clinical trials include the strict contraceptive requirements and concerns for the safety of the embryo and foetus. Women may also be concerned about the outcome of future pregnancies after participating in such trials (5). The partners of female trial participants may refuse sexual intercourse due to the woman's involvement in a trial. Furthermore, a lack of convenient clinic hours for mothers, domestic workers and sex workers (3) presents an additional barrier to women's participation. In many cultures, women are not able to make decisions to participate in such trials without their spouse's permission $(4,5)$.

Enrolment in AIDS vaccine trials by both men and women may also be hindered by public misunderstanding about the risk of contracting HIV from candidate vaccines. Furthermore, a falsepositive antibody test could lead to a participant being mistakenly viewed as HIV-infected, and facing social stigma and discrimination as a consequence.

Concerns about unknown effects of any investigational agent on an embryo and foetus, and potential risks to the future reproductive capacity of female participants (9) demands that pregnant women beexcluded from enrolling into trials of investigational new drugs, and women of reproductive potential are expected to use contraception during such trials (10). AIDS candidate vaccine trials are no exception. Thus, occurrence of pregnancies among female participants in AIDS candidate vaccine clinical trials further reduces female participation by reducing the number of women from whom complete information about a candidate vaccine can be obtained, since vaccination has to be stopped when a pregnancy occurs. The issue of contraception thus needs to be addressed if meaningful data from female participants in such trials are to be obtained. Otherwise, high pregnancy rates should be taken into consideration when the sample size is being determined (10).

In our trials, the female participants were mostly young, single and in the reproductive age group. The requirement to avoid pregnancy during a specified period presented a challenge, even with regular family planning counselling prior to enrolment and during each protocol visit. The situation is compounded by the fact that many women in developing-countries settings are not in a position to choose a contraceptive method without their partner's permission.

A previous review of safety data from 25 Phase I and Phase II AIDS vaccine clinical trials involving low-risk female participants found a pregnancy rate of $2.9 \%$ (11). The higher pregnancy rate at our site may be attributable to the fact that female participants at our site were younger than those reported in this review. Additionally, the higher pregnancy rate seen in our trials compared to that in the reported studies may have been due to differences in contraception methods used by our female participants and those in the studies reviewed. Some of the participants in our trials used sexual abstinence as their preferred contraceptive method, while in the reviewed studies abstinence was not an approved contraceptive option.

Ahigh pregnancy incidence has also been noted among high-risk women participating in a HIV vaccine efficacy trial (9). In this trial lasting three years, pregnancy incidence was calculated on the basis of person-years of follow-up. Published preliminary pregnancy incidence over the first 18 months of this study was 4.6 per 100 person years (9). Those who became pregnant were more likely to be less than 35 years and at baseline reported unprotected sex in the previous six months. It may however be expected of women at high risk of acquiring HIV to also have a higher pregnancy rates therefore negating the comparison of the rates in populations with different risks of HIV acquisition.

All the women whobecame pregnant in our trials were single and mostly primigravida. They had all 
elected to use sexual abstinence as a contraception method, but evidently engaged in unprotected sex. Although we did not see pregnancies among the two participants who reported consistent use of male condoms by their sexual partners, this observation may be due to the small sample size. Male condoms usage is likely to have drawbacks similar to abstinence, as their effective use largely depends on the co-operation of the sexual partners. The use of effective female-controlled contraceptives in trials would go along way towardsensuring that all females enrolled complete the trial, and that sufficient data are gathered to allow quantitative analyses.

In conclusion, our data indicate that despite repeated family planning counselling, female participants in AIDS candidate vaccine clinical trials in Kenya experienced difficulty in preventing pregnancy when their chosen family planning method was sexual abstinence even though they were single. We recommend therefore that sexual abstinence should not be allowed as an option in future clinical trials.

Efficacious and long-acting female-controlled contraceptive methods, administered at the clinical trial site, should be offered instead.

\section{ACKNOWLEDGEMENTS}

We thank all the volunteers who participated in these trials. We also thank the staff at KAVI for the splendid work done and Daisy Ouya for reviewing the manuscript and making valuable comments and suggestions. All the AIDS vaccine clinical trials at KAVI were funded by the International AIDS Vaccine Initiative (IAVI). IAVI 010 trial was conducted jointly with St. Thomas Hospital in London, whileIAVIVRC V001 trial was conducted jointly with Project San Francisco (PSF) site in Kigali, Rwanda.

Conflict of Interest: The authors Patricia Fast, Claudia Schmidt and Carl Verlinde are either employees or consultants of IAVI, the funding organisation and have no commercial interest or other association that might raise a conflict of interest.

\section{REFERENCES}

1. Rudy, E.T., Newman, P.A., Duan, N. et al.HIV vaccine acceptability among women at risk: Perceived barriers and facilitators to future HIV vaccine uptake. AIDS Education and Prevention. 2005; 17:253-267.

2. UNAIDS/WHOJoint United Nations Programme on HIV / AIDS. AIDS epidemic update: UNAIDS 2007, Geneva, Switzerland. http://www.unaids.org.

3. Mills, E., Nixon, S., Singh, S.et al.Enrolling women into HIV preventive vaccine trials: an ethical imperative but a logistical challenge. PloS Medicine. 2006; 3:3 e94.

4. WHO-UNAIDS Expert Group. Gender, age, and ethnicity in HIV vaccine-related research and clinical trials, report from a WHO-UNAIDS consultation, 2628 August 2004. Lausanne, Switzerland. AIDS. 2005; 19:w7-28.

5. UNAIDS/WHO (2007b) Ethical consideration in biomedical HIV prevention trials. UNAIDS(2007). http://data.unaids.org/pub/Reportl2007/iG1399-eth icalconsiderations en.pdf.

6. Sheon, A.R., Wagner, L., McElrath, M.J., et al. Preventing discrimination against volunteers in prophylactic HIV vaccine trials. Lessons from a phase II trial. J. Acq. Immuno. Syndr. Hum. Retr. Infect. 1998; 19:519-526.

7. Buchbinder, S.P., Mehrotra, D.V., Duerr, A., et al. Step Study Protocol Team. Efficacy assessment of a cellmediated immunity HIV-1 vaccine (the Step Study): a doubleblind, randomised, placebo-controlled, testof-concept trial. Lancet. 2008; 372: 1881-1893.

8. Kiwanuka, N., Robb, M., Kigozi, G., et al. Knowledge about vaccines and willingness to participate in preventive HIV vaccine trials: a population-based study, Rakai, Uganda. J. Acq. Immune. Defic. Syndr. 2004; 36:721-725.

9. Brown-Peterside, P., Ren, L., Hirsch, A., et al and the Vaxgen Study Team Pregnancies, among high-risk women in the first ever HIV vaccine efficacy trial. AIDS. 2003; 17: 787-788.

10. Bennett, J.C. Inclusion of women in clinical trials policies for population subgroups. New Engl. J. Med. 1993; 329:288-292.

11. Keefer, M.C., Wolff, M., Gorse, G.J., et al. and the NIAD AIDS Vaccine Evaluation Group. Safety profile of phase I and II preventive HIV type envelope vaccination: experience of the NIAD AIDS Vaccine Evaluation Group. AIDS Res. Human Retroviruses. 1997; 13:1163-1177. 